\title{
Caracterización cuantitativa y cualitativa de evidencias de aprendizaje por parte de público escolar en la exposición itinerante «Animales de la Amazonía: conocer para preservar»
}

\author{
Yonier Alexander Orozco Marin ${ }^{1,2, a}$, Patrícia Santos Ferreira-Peruquetti ${ }^{3}$, Yuri Karaccas \\ de Carvalho ${ }^{3}$ \\ ${ }^{1}$ Mestrado profissional em Ensino de Ciências e Matemática, Universidade Federal do Acre. Rio Branco. \\ Acre. Brasil. \\ ${ }^{2}$ Grupo de Investigación Biología, Enseñanza y Realidades. Universidad Distrital Francisco José de Caldas. \\ Bogotá. Colombia. \\ ${ }^{3}$ Centro de Ciências Biológicas e da Natureza - CCBN, Universidade Federal do Acre. Rio Branco. Acre. \\ Brasil. \\ aapmusicomano@gmail.com
}

[Recibido: 1 Enero 2018. Revisado: 23 Marzo 2018. Aceptado: 5 Julio 2018]

\begin{abstract}
Resumen: El objetivo de este trabajo fue el de caracterizar las evidencias de aprendizaje sobre el tema «Vertebrados de la Amazonía» por parte de público escolar de una escuela pública de la ciudad de Rio Branco (Acre, Brasil) en la exposición itinerante «Animales de la Amazonía: Conocer para preservar». A través de la comparación de parámetros cuantitativos y cualitativos en los mapeos de significado personal (PMM, por sus siglas en inglés) realizados por los estudiantes antes y después de la visita. Tres parámetros fueron analizados cuantitativa y cualitativamente en los PMM: extensión, alcance y profundidad. El aprendizaje de los alumnos se caracterizó por un aumento en el vocabulario utilizado para referirse al tema «Vertebrados de la Amazonía», el abordaje del asunto desde nuevas y diferentes perspectivas, con un énfasis mayor en la perspectiva ecológica. Los resultados destacan el potencial de las exposiciones itinerantes para contribuir al aprendizaje del público escolar sobre la biodiversidad local y sugieren la necesidad de investigar los procesos de evaluación del impacto en los visitantes de estos espacios.
\end{abstract}

Palabras clave: Espacios no formales de educación; Mapeo de significado personal; Evaluación del aprendizaje; Exposición itinerante.

Quantitative and qualitative characterization of learning evidences by the school public in the itinerant exhibition "Animals of the Amazon: Knowing to preserve"

Abstract: Students are frequently interested in Amazon rainforest fauna. This study is aimed to investigate evidences of high school students learning outcomes from the itinerant exhibition "Amazon Animals: Knowing to preserve", in Rio Branco municipality (Acre, Brazil), through the comparison of parameters in Personal Meaning Mapping (PMM). The PMM parameters Extension, Scope and Depth were analyzed (before and after visits) quantitative and qualitatively. Students' learning was characterized by an increase in vocabulary to refer to "Amazonian vertebrates", with a greater emphasis on ecological perspective. Our results highlight the potential of traveling exhibitions to contribute to the greater public learning about local biodiversity and suggest the need to investigate the evaluation processes on visitors to these spaces.

Keywords: Non-formal educational spaces; Personal Mapping Meaning; Learning evaluation; Mobile exhibition.

Para citar este artículo: Orozco Marin Y. A., Ferreira-Peruquetti P. S., Carvalho Y. K. (2018) Caracterización cuantitativa y cualitativa de evidencias de aprendizaje por parte de público escolar en la exposición itinerante «Animales de la Amazonía: conocer para preservar». Revista Eureka sobre Enseñanza y Divulgación de las Ciencias 15 (3), 3106. doi: 10.25267/Rev_Eureka_ensen_divulg_cienc.2018.v15.i3.3106 


\section{Introducción}

El bioma amazónico ocupa un área de aproximadamente siete millones de kilómetros cuadrados y está localizado al norte de América del Sur (Menin 2010). Esta región se destaca por presentar uno de los índices de diversidad biológica más elevados del planeta. Este aspecto llama la atención de científicos, naturalistas y conservadores de la biodiversidad en todo el mundo.

Dentro de toda esa diversidad biológica, se encuentra uno de los grupos taxonómicos más conocidos. Se trata de los vertebrados. Según Roland y Vasconcelos (2007), el conocimiento sobre este grupo es mayor que el de otros organismos e, inclusive, muchos de los endemismos registrados en Brasil se encuentran en la región amazónica. Este mayor conocimiento se debe a que muchas de las prácticas culturales, espirituales y económicas de la región se encuentran asociadas a estos organismos. Sin embargo, muchas de esas prácticas están afectando negativamente la diversidad biológica.

Acciones como la deforestación acelerada, el crecimiento urbano y la expansión agrícola, ocasionan alteraciones en los ecosistemas amazónicos, que pueden desencadenar disminución de los índices de diversidad de los vertebrados silvestres. Este aspecto sugiere la necesidad de fortalecer procesos educativos que aborden la temática de las características de los vertebrados silvestres y las posibilidades para su conservación.

Particularmente, en las regiones urbanas de la Amazonía, el contacto de los sujetos de los contextos escolares con el bosque es menor que en contextos rurales. A pesar de que los bosques están relativamente cerca de la ciudad, son pocos los espacios institucionalizados y con instalaciones adecuadas para que los profesores planeen actividades en espacios naturales.

En ese contexto, la Exposición itinerante «Animales de la Amazonía: conocer para preservar», se destaca como la primera en la ciudad de Rio Branco (Acre, Brasil) en tratar asuntos relacionados con la diversidad de vertebrados silvestres de la Amazonía en contextos escolares. Según Schwenck (2011), las exposiciones itinerantes contribuyen a la divulgación científica, facilitando el acceso de las poblaciones más distantes, física e intelectualmente, a la cultura científica. Este trabajo parte de la consideración de que estos espacios también pueden, y deben, adaptarse a distintos contextos educativos, socioeconómicos y culturales. Construyendo nuevos significados, no siempre científicos, al lado de los sujetos que participan del proceso de enseñanza y de aprendizaje en el contexto donde la exposición es presentada.

Rocha y Marandino (2017) destacan que son diversos los desafíos de los espacios itinerantes para desarrollar actividades educativas en el contexto brasileño. El primero consiste en el apoyo financiero y político. El segundo se refiere a los recursos humanos, las relaciones con otras instituciones y los modelos de comunicación científica. Por último, se encuentra el desafío de la investigación sobre los procesos de enseñanza y aprendizaje en estos espacios y la evaluación de su impacto.

Esta investigación aborda este último desafío, específicamente, la evaluación de los aprendizajes de los sujetos en la exposición. Considerando el tiempo breve de interacción de la visita y la heterogeneidad de perspectivas y expectativas que los visitantes otorgan a la experiencia. Estos aprendizajes han sido caracterizados con diversas metodologías, tanto cualitativas como cuantitativas, dependiendo de los intereses del investigador y de la concepción de aprendizaje que fundamentó la investigación (Bizerra y Marandino 2009).

Marandino et al. (2008) mencionan que los aprendizajes alcanzados por el público escolar en los espacios educativos no formales deben ser estimulados por medio de negociaciones activas 
entre visitante, mediador y los objetos de la exposición. Esta consideración coincide con el planteamiento de la tridimensionalidad del aprendizaje (Falk y Dierking 2002). Este planteamiento considera el aprendizaje como un fenómeno tridimensional que surge de la combinación de planos personales, sociales y físicos.

En esta investigación se entendieron las evidencias de aprendizajes de un visitante como muestras de un proceso que es diferente en cada sujeto y que se nutre del conjunto de preconceptos, conocimientos, actitudes y valores del sujeto antes de la visita (plano personal). Ese aprendizaje también se nutre de las interacciones dialógicas que el visitante establece durante la visita con mediador, objetos de la exposición y otros visitantes (plano social). Proceso que es favorecido por los materiales didácticos y por la disposición de los objetos que invitan a la exploración y a la participación activa en la exposición (plano físico). En ese sentido, se considera que la evaluación de los aprendizajes de los visitantes en una exposición itinerante debe permitir la caracterización de aspectos del contenido de esos aprendizajes, pero también del proceso de cómo fueron construidos.

Falk y Storksdieck (2005) proponen los Personal Meaning Mapping (PMM, mapeos de significado personal) como instrumentos de evaluación en espacios no formales de educación. Los PMM constituyen una herramienta que, cuando se planifica y orienta adecuadamente, permite reconocer el aprendizaje del alumno como un proceso que parte de sus propios conocimientos y no únicamente como un punto final de llegada uniforme para todos. Los PMM presuponen que no existe un único resultado válido de aprendizaje; sí existen diversas posibilidades que vinculan las ideas de los propios alumnos, sus intereses y los significados que construyen durante la experiencia en la visita.

El objetivo de este trabajo fue caracterizar las evidencias de aprendizaje sobre los vertebrados de la Amazonía por parte de 82 estudiantes del primer año (rango de edad: 14-19 años) de la Enseñanza Media de una escuela pública de la ciudad de Rio Branco (Acre, Brasil), en la Exposición itinerante «Animales de la Amazonía: conocer para preservar». A través de la comparación de parámetros cuantitativos y cualitativos en los Personal Meaning Mapping (PMM) realizados por los estudiantes antes y después de la visita.

\section{Metodología}

El marco metodológico que orientó esta investigación consistió en la investigación mixta, que consiste en la combinación de técnicas, métodos, enfoques, conceptos, y lenguaje cualitativo y cuantitativo en un único estudio (Johnson y Onwuegbuzie 2004) Con el fin de obtener una comprensión más amplia sobre el objeto de estudio (Creswell y Garret 2008), en este caso, las evidencias de aprendizaje sobre el tema vertebrados de la Amazonía por parte de público escolar, después de una visita a la Exposición itinerante «Animales de la Amazonía: conocer para preservar».

La exposición «Animales de la Amazonía: conocer para preservar» es una propuesta educativa del Laboratorio de Anatomía Animal de la Universidad Federal del Acre. La exposición cuenta con piezas (esqueletos) de animales vertebrados del bioma amazónico, que son seleccionados y dispuestos de acuerdo a la temática de cada presentación. El tema de la exposición fue la alimentación de los animales de la Amazonía y la relación con las estructuras anatómicas. Se abordaron las características morfológicas relacionadas con la alimentación de los animales de la exposición (captura de alimento, masticación, etc.), la posición en la cadena alimentaria y las posibles relaciones alimentarias entre los animales de la exposición.

El mono barrigudo y la jiboia (boa) formaron parte de una exposición en 2015 (Peruquetti et al. 2018), cuyo objetivo fue mostrar técnicas de estudio de anatomía y la preparación de 
colecciones biológicas. Las otras piezas (tabla 1) fueron montadas en los años siguientes como parte del proyecto que dio nombre a la exposición «Animales de la Amazonía: conocer para preservar». La colección aumenta su número de piezas, con proyectos de montaje de esqueletos de animales silvestres en asociación con instituciones gubernamentales que donan cadáveres de animales incautados del tráfico ilegal, con fines didácticos y científicos. Las figuras 1 y 2 muestran ejemplos de algunas de las piezas utilizadas en la exposición.

Tabla 1. Esqueletos presentados en la exposición itinerante «Animales de la Amazonía: conocer para preservar». Se han mantenido los nombres autóctonos de los animales tal como se usan en la región estudiada.

\begin{tabular}{|l|l|l|}
\hline $\begin{array}{l}\text { Nombre científico y nombre } \\
\text { común en la Amazonía brasileña } \\
\text { junto a la denominación local, aparece el } \\
\text { nombre en castellano) }\end{array}$ & Grupo taxonómico & Hábito alimentacio \\
\hline $\begin{array}{l}\text { Arapaima gigas } \\
\text { Pirarucu - Arapaima }\end{array}$ & Pez osteoglossiforme & Omnívoro \\
\hline $\begin{array}{l}\text { Boa sp. } \\
\text { Jiboia - Boa }\end{array}$ & Réptil & Carnívoro \\
\hline $\begin{array}{l}\text { Buteo nitidus } \\
\text { Gavião pedrês - Gavilán saraviado }\end{array}$ & Ave & Carnívoro \\
\hline $\begin{array}{l}\text { Hydrochoerus hydrochaeris } \\
\text { Capivara - Capibara }\end{array}$ & Mamífero & Herbívoro \\
\hline $\begin{array}{l}\text { Lagotbrix lagotricha } \\
\text { Macaco barrigudo }\end{array}$ & Mamífero & Omnívoro \\
\hline $\begin{array}{l}\text { Mitu tuberosum } \\
\text { Mutum - Paujil }\end{array}$ & Ave & Omnívoro \\
\hline $\begin{array}{l}\text { Panthera onca } \\
\text { Onça - Jaguar }\end{array}$ & Mamífero & Carnívoro \\
\hline $\begin{array}{l}\text { Priodontes maximus } \\
\text { Tatu canastra - Armadillo gigante }\end{array}$ & Mamífero & Insectívoro \\
\hline
\end{tabular}

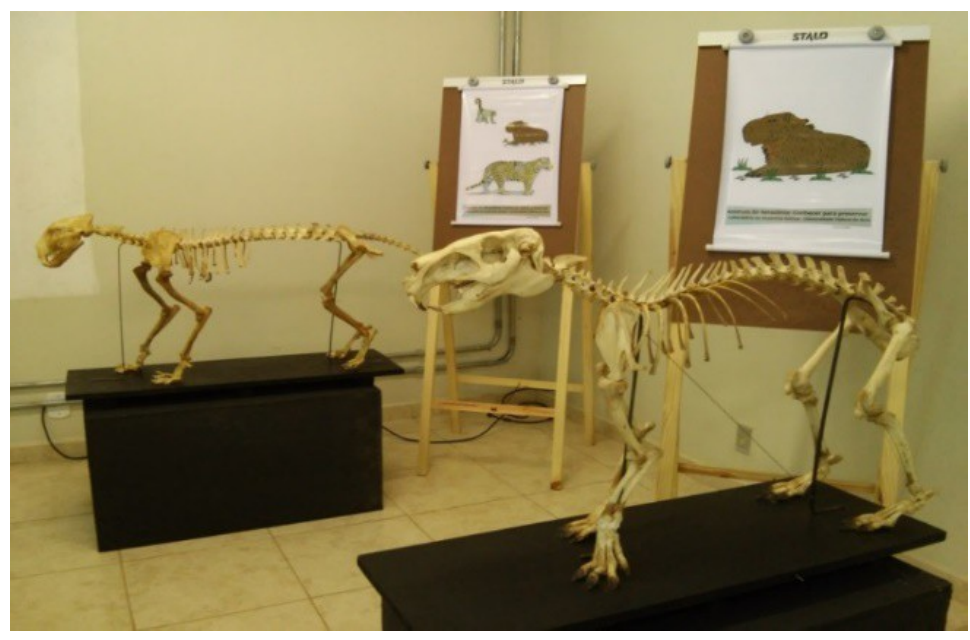

Figura 1. Esqueletos completos y montados de onça y capivara de la exposición «Animales de la Amazonía: conocer para preservar.»

Las actividades de enseñanza y aprendizaje durante la visita se dividieron en dos partes. La primera consistió en la observación de los esqueletos, mediada por los monitores de la exposición a través del diálogo y la exploración. La segunda parte consistió en la realización de actividades lúdicas e interactivas en un área del bosque del Parque Zoobotánico de la Universidad Federal del Acre. 
Las dos partes de las actividades se fundamentaron en la propuesta de recorridos guiados indagatorios en museos (Roldan et al. 2017), que comprende tres elementos esenciales para vincular activamente al visitante en la experiencia: a) La pregunta como estrategia para captar la atención del visitante; b) orientaciones sobre las acciones que el visitante debe realizar para garantizar la exploración de los objetos, y la interacción desde diferentes sentidos, y c) la estimulación de reflexiones sobre lo observado por parte del visitante.

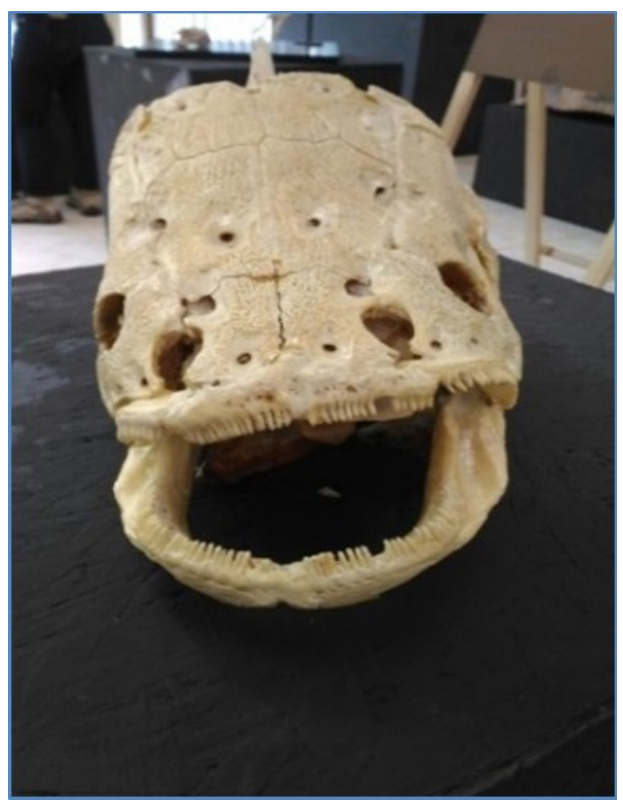

Figura 2. Cráneo de pirarucu en la exposición «Animales de la Amazonía: conocer para preservan».

Los sujetos participantes de la investigación correspondieron a 82 alumnos (46 alumnas, 36 alumnos) distribuidos en cuatro clases del primero año de la Educación Media de una escuela estatal de la Ciudad de Rio Branco (Acre, Brasil). El promedio de edad de los alumnos fue 15 años (rango 14-19 años). Se estableció un acuerdo ético con los alumnos y sus responsables legales a través de la firma de término de libre consentimiento para el desarrollo de la investigación, asegurando el uso de los datos para fines estrictamente relacionados a los objetivos de la investigación.

Los alumnos participaron en las actividades de la exposición itinerante, durante cuatro días. Cada día se recibió la visita de un curso. Los cuatro cursos participaron de los dos momentos de las actividades de la exposición. Las actividades acontecieron entre el 9 y el 12 de mayo de 2017, pues son fechas donde disminuyen las lluvias en la región, facilitando el contacto de los alumnos con el bosque. El número de alumnos de cada curso se describe en la tabla 3 .

Como instrumento de recogida de datos se utilizó la estrategia del Personal Mapping Meaning adaptado de la propuesta de Falk y Storksdieck (2005), Delicado et al. (2010) y Villa (2016). Es una derivación del mapeo de conceptos, en la que a los encuestados primero se les pidió una lluvia de ideas sobre una palabra o expresión. El PMM es una herramienta que permite identificar el cambio de percepción o conocimientos sobre un asunto específico durante una visita. Es una tarea abierta que ofrece la posibilidad al visitante de expresarse en la manera que le resulte más fácil, y a partir de la cual pueden ser analizados diversos aspectos de acuerdo a los intereses del investigador.

Antes de la visita se entregó a cada alumno una hoja dividida en dos partes. La primera constaba de cuatro preguntas básicas sobre el nombre del alumno, sexo, edad y curso (a, b, c, d). La segunda consistía en el PMM. En la parte central de la hoja se encontraba la frase 
«Vertebrados de la Amazonía». Se dieron a los alumnos las siguientes indicaciones: a) Piense en todo lo que viene a su mente cuando escucha el término «Vertebrados de la Amazonía»; b) Utilice la hoja para expresar de manera escrita, o a través de mapas conceptuales, esas ideas que vienen a su mente sobre el tema, y c) Por favor, recuerde que no se trata de un test de respuestas únicas, o respuestas correctas y erróneas, simplemente queremos conocer su percepción y conocimiento sobre el asunto.

Se realizó un registro fotográfico de los PMM de cada alumno y se asignó un código aleatorio a cada uno. Los alumnos participaron en las dos partes de las actividades de la exposición, con una duración aproximada de 120 minutos. Una vez finalizada la visita, se entregó a cada alumno el mismo PMM que había realizado antes de la visita, dando las siguientes indicaciones: a) Por favor, lea las ideas que usted expresó sobre la temática «Vertebrados de la Amazonía»; b) Considerando su experiencia durante la exposición, siéntase libre de modificar, ratificar, retirar, o añadir los aspectos sobre el tema que fueron significativos para usted. Nuevamente se realizó un registro fotográfico de los PMM para facilitar la comparación entre las respuestas antes y después de la visita.

El estudio se basó en un diseño de medidas repetidas que incluía evaluación previa y posterior. Los análisis fueron realizados sobre tres parámetros que permiten obtener una comprensión general y amplia del aprendizaje de los alumnos, a partir de la propuesta de análisis de los PMM de Falk y Storksdieck (2005). Para procedimientos de análisis identificamos como PMM-Pre (los PMM realizados por los alumnos antes de la visita) y como PMM-Pos (los PMM realizados después de la visita) se realizó un análisis cualitativo y cuantitativo de las respuestas de los alumnos antes y después de la visita.

Según Falk y Storksdieck (2005), el cambio en la conceptualización de un individuo en el PMM puede «cuantificarse» mediante cuatro parámetros: extensión, alcance, profundidad y dominio. Los autores definen los cuatro parámetros tal como se describe a continuación.

Extensión se refiere a cambios en el número de palabras que se utilizan para describir el tema, indicando un incremento en el vocabulario que un individuo utiliza para describir un fenómeno o asunto. La comprensión de cualquier tema pasa por el uso del lenguaje para describir esa comprensión, por lo tanto, extensión se trata de un aspecto básico para caracterizar el aprendizaje de un sujeto.

El alcance (parámetro también denominado amplitud), trata del cambio en el número de categorías conceptuales o perspectivas que utiliza un individuo para comprender un tema. Este parámetro contempla que un mismo asunto puede ser abordado desde diferentes perspectivas, y que una experiencia de aprendizaje puede favorecer otros abordajes en los sujetos.

La profundidad trata del cambio en el número de palabras que el individuo utiliza para describir el tema dentro de cada perspectiva o categoría conceptual de ese tema. Un aumento en ese parámetro puede representar una comprensión conceptual más profunda y elaborada dentro de una categoría conceptual o perspectiva particular. El último parámetro se refiere al dominio. Falk y Storksdieck (2005) los describen como una medida más tradicional del aprendizaje que pretende «medir» el nivel de dominio general (que puede ir de principiante a experto) de un sujeto en relación al tema propuesto en el PMM.

Antes de especificar cómo se analizaron los datos, es importante realizar dos aclaraciones. La primera, se trata de que en este trabajo no se analizó el parámetro dominio, pues se parte del presupuesto de que en la Amazonía, además del conocimiento científico-académico, existe una variedad de perspectivas y conocimientos culturales asociados a la diversidad biológica, y puede ser un problema ético establecer medidas para caracterizar un nivel de «principiante» $\mathrm{O}$ de «experto». La segunda aclaración se trata de que la propuesta de Falk y Storksdieck (2005) 
no contempla el análisis cualitativo de los parámetros. Sin embargo, en este trabajo se optó por realizar un análisis cualitativo que permita identificar aspectos más relacionados con el proceso de aprendizaje, y no únicamente centrados en el contenido del PMM como un producto acabado.

\section{Análisis cuantitativo de los parámetros}

En relación al parámetro extensión, primeramente se realizó el conteo de las palabras. Fueron contabilizadas todas las palabras mencionadas por los alumnos, incluyendo las preposiciones, conjunciones, interjecciones, artículos y pronombres. En el caso de sustantivos, verbos o adjetivos repetidos en una misma oración, estos se contabilizaron como uno. Ejemplo: «El Pirarucu come peces pequeños y come frutas». En este caso, la palabra come fue contabilizada una sola vez.

El análisis estadístico para el parámetro extensión consistió en comparar el número de palabras mencionadas por los alumnos de cada clase antes y después de la visita (prueba no paramétrica, muestras independientes) utilizando la prueba Kruskal-Wallis (1952).

Para el parámetro alcance, primeramente se clasificaron las palabras mencionadas por los alumnos en categorías o perspectivas de análisis del tema de la exposición. Las perspectivas (reconocimiento, anatómico, ecológico y cultural) fueron establecidas después del análisis cualitativo de los PMM, de acuerdo al procedimiento descrito más adelante. Se contabilizó y comparó el número de perspectivas que los alumnos abordaron antes de la visita, con el número de perspectivas abordadas después de la visita.

Posteriormente, para el parámetro extensión se comparó la media de las palabras mencionadas por los alumnos dentro de cada perspectiva (prueba no paramétrica, muestras dependientes) por la prueba de Friedman (1940). Tanto las pruebas con análisis de varianza como con datos medidos a nivel ordinal, son adecuadas para el análisis por categorías-perspectivas como propuesto.

La comparación de las respuestas de los alumnos antes y después de la visita en la exposición se dio con el intervalo de confianza del 95\%. La no superposición de las barras representativas del intervalo de confianza indica una diferencia significativa (Belia et al. 2005, Cumming et al. 2007). El programa estadístico utilizado para pruebas y gráficos fue StatSoft, Inc. (2014). STATISTICA (Data Analysis Software System), versión 12, www.statsoft.com.

\section{Análisis cualitativo de los parámetros}

Cualitativamente, el parámetro extensión fue considerado como la relación que el alumno estableció (o no) entre lo mencionado en el PMM antes de la visita y el PMM después de la visita. La relación fue identificada y clasificada como relación de continuidad o de discontinuidad. Para ser identificada como relación de continuidad se analizó la presencia de alguno de estos elementos en los PMM-Pos: a) flechas conectando frases o palabras escritas antes y después de la visita; b) frases o palabras completando frases o palabras que fueron mencionadas antes de la visita; c) palabras o frases que fueron corregidas o reemplazadas por palabras o frases nuevas. Cuando no fueron identificados ninguno de estos elementos, la relación se clasificó como discontinuidad. De esta manera, sobre el parámetro extensión, además de caracterizar el cambio en relación a la cantidad de palabras expresadas, también se identificó la relación que el alumno estableció entre las nuevas informaciones a las que tuvo acceso en la visita, con su conocimiento previo.

En relación al parámetro alcance, primeramente se identificaron y separaron todas las palabras individuales, frases cerradas o parágrafos mencionadas en cada PMM. Posteriormente, se leyeron y analizaron todas las frases de los PMM de todos los alumnos. Las frases se 
agruparon de acuerdo al eje temático que abordaban sobre los vertebrados de la Amazonía. Estos ejes temáticos fueron identificados como perspectivas de explicación y utilizados para el análisis cuantitativo del parámetro alcance y profundidad.

En la lectura de esas frases en los PMM-Pre, se realizó un análisis de dos aspectos que son relevantes sobre el conocimiento previo de los alumnos, en relación a la diversidad de vertebrados de la Amazonía: a) la mención de animales vertebrados nativos o exóticos; b) la identificación de ideas equivocadas sobre los vertebrados desde una perspectiva científica.

Cualitativamente, el parámetro profundidad fue comprendido como el nivel de pensamiento alcanzado por los alumnos antes y después de la visita. Según Webb et al. (2005), el nivel de pensamiento consiste en el conjunto de habilidades cognitivas que el sujeto utiliza en la construcción de enunciados y descripción de un tema específico (repetición, conceptual, pensamiento estratégico, pensamiento extendido). Estas habilidades pueden ir desde la mera reproducción de informaciones y datos en frases cortas, hasta la construcción de frases y párrafos que evidencian la conexión entre diversas informaciones para construir un argumento o explicación.

Para la temática «Vertebrados de la Amazonía» y considerando las características de la exposición, se definieron los niveles de pensamiento descritos en la tabla 2.

Tabla 2. Niveles de pensamiento en el abordaje de la temática «Vertebrados de la Amazonía». Adaptado de Webb et al. (2005).

\begin{tabular}{|l|l|l|}
\hline $\begin{array}{l}\text { Nivel de } \\
\text { pensamiento }\end{array}$ & Acciones del sujeto en los PMM & Ejemplos \\
\hline $\begin{array}{l}\text { Nivel 1: } \\
\text { Repetición }\end{array}$ & $\begin{array}{l}\text { Reproducción de informaciones, datos, } \\
\text { definiciones o procedimientos simples } \\
\text { sobre los animales de la exposición. }\end{array}$ & $\begin{array}{l}\text { kLa capivara come pasto» } \\
\text { 《El jaguar tiene los ojos al frente del cráneo» }\end{array}$ \\
\hline $\begin{array}{l}\text { Nivel 2: } \\
\text { Conceptual }\end{array}$ & $\begin{array}{l}\text { Requiere uso de habilidades mentales, } \\
\text { clasifica y agrupa por características en } \\
\text { común los animales de la Exposición y } \\
\text { utiliza terminología de conceptos para } \\
\text { describirlos. }\end{array}$ & $\begin{array}{l}\text { kLa capivara es un ejemplo de animal herbívoro, } \\
\text { pues su dieta consiste en material vegetal, por } \\
\text { ejemplo, el pasto. » }\end{array}$ \\
\hline $\begin{array}{l}\text { Nivel 3: } \\
\text { Pensamiento } \\
\text { estratégico }\end{array}$ & $\begin{array}{l}\text { Establece conexiones evidentes entre } \\
\text { perspectivas de explicación de los } \\
\text { vertebrados de la Amazonía aprovechando } \\
\text { las características observadas en los } \\
\text { esqueletos. }\end{array}$ & $\begin{array}{l}\text { kEl jaguar es un ejemplo de animal carnívoro al } \\
\text { igual que el gavilán. Ambos están } \\
\text { anatómicamente adaptados para el consumo de } \\
\text { carne, generalmente proveniente de presas vivas. } \\
\text { Por eso tienen los ojos ubicados hacia el frente, } \\
\text { pues eso les permite tener una mayor } \\
\text { profundidad en la visión para localizar su presa.» }\end{array}$ \\
\hline $\begin{array}{l}\text { Nivel 4: } \\
\text { Pensamiento } \\
\text { extendido }\end{array}$ & $\begin{array}{l}\text { Muestra esfuerzos cognitivos evidentes } \\
\text { por integrar las diferentes perspectivas de } \\
\text { explicación dos vertebrados con otros } \\
\text { campos del conocimiento, construyendo } \\
\text { nuevas ideas que no fueron repasadas en la } \\
\text { exposición. }\end{array}$ & $\begin{array}{l}\text { kLa manera en que las características anatómicas } \\
\text { del jaguar están adaptadas para la depredación, } \\
\text { puede ser entendida como el producto de un } \\
\text { proceso evolutivo en la floresta Amazónica, que } \\
\text { permitió la selección y formación de un fenotipo } \\
\text { aracterizado por la disposición de los ojos al } \\
\text { frente, una alta profundidad en la visión, garras } \\
\text { especializadas...» }\end{array}$ \\
\hline
\end{tabular}

Se identificaron y separaron todas las frases o parágrafos de cada PMM antes y después de la visita. Cada frase o parágrafo se analizó según los cuatro niveles de pensamiento establecidos. Cuando las frases o parágrafos de un mismo PMM tuvieron clasificaciones en niveles diferentes, se le asignó a todo el PMM la clasificación del nivel más alto alcanzado en alguna de las frases o parágrafos. En el caso de los PMM en los cuales no se identificaron frases y que 
únicamente fueron mencionadas palabras aisladas (generalmente el nombre común de vertebrados de la Amazonía), estos fueron clasificados como repetición.

\section{Resultados y discusión}

A continuación presentamos los resultados y análisis cuantitativos y cualitativos para cada parámetro caracterizado en los PMM realizados por los alumnos antes y después de la visita.

\section{Extensión}

No hubo diferencia significativa en el número de palabras mencionadas entre los alumnos de las cuatro clases tanto en el PPM-Pre (Kruskal-Wallis $(3, n=82) H=0,75689, p=0,8597)$, como en el PMM-Pos (Kruskal-Wallis (3, $n=82) H=5,3927, p=0,1452)$. A partir de estos resultados (figura 3), podemos considerar que antes y después de la visita los alumnos de las cuatro clases tuvieron un comportamiento homogéneo en relación a la cantidad de palabras utilizadas para referirse al eje temático de la exposición. Con este resultado no probamos si hubo diferencia de edad y género. Sin embargo, hubo un aumento significativo del número de palabras en el PMM después de la visita a la exposición. La clase A tuvo un aumento del 10,85\%, la clase B $41,86 \%$, la clase C 37,13 \% y la clase D 27,68 \%, con un aumento medio total del $30 \%$ considerando las cuatro clases (tabla 3 ).

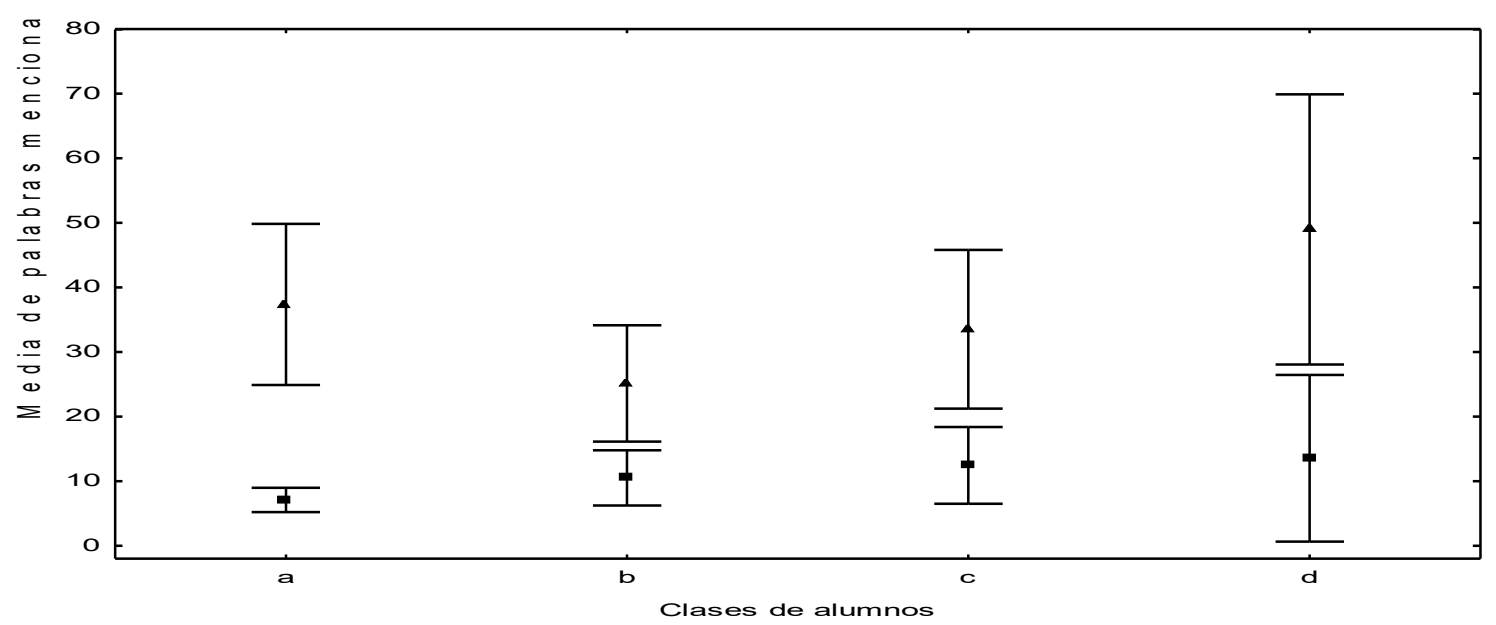

Figura 3. Media de palabras mencionadas por cada clase de alumnos (A, B, C, D) participantes en el PMM-Pre (cuadrados llenos) y en el PMM Pos (triángulos). Las diferencias no fueron estadísticamente significativas entre los grupos de alumnos (Kruskal-Wallis pre $(3, n=82) H=0,75689, p=0,8597)$, Kruskal-Wallis pos $(3 ; n=82) H=$ 5,3927, $p=0,1452$, pero fueron significativas entre el PMM-Pre y el PMM-Pos. La no superposición de las barras representativas del intervalo de confianza de $95 \%$ indica una diferencia significativa.

Los resultados cuantitativos en este parámetro coinciden con otras investigaciones que utilizaron PMM para caracterizar evidencias de aprendizajes en espacios no formales. Sanches, Leite y Leroy (2011) reportaron que en términos de cantidad de palabras, nuevos términos fueron adquiridos por los alumnos después de la visita a un planetario. Moss, Jensen y Gusset (2014) también encontraron que en 26 zoológicos de 19 países el entendimiento de los visitantes sobre lo que es biodiversidad también aumentó. Ese aumento está relacionado con lo que Leinhardt, Knuston y Crowley (2002) denominan como un enriquecimiento del vocabulario a través de la elaboración conversacional estimulada por el dialogo en las actividades realizadas durante la visita. Ese aumento se comprende como producto de las interacciones, diálogos, observaciones y experiencias durante la visita que permiten que el alumno tenga acceso a nuevas informaciones que pueden considerar importantes para reproducir o integrar a sus estructuras conceptuales previas. 
Tabla 3. Porcentaje de aumento de palabras mencionadas por los alumnos en el PMM antes y después de la visita a la exposición «Animales de la Amazonía: conocer para preservar». El número total de alumnos es $n$.

\begin{tabular}{|c|c|c|c|c|c|c|}
\cline { 3 - 6 } \multicolumn{2}{c|}{} & \multicolumn{3}{c|}{ Antes } & \multicolumn{2}{c|}{ Después } \\
\hline Clase & $n$ & Promedio & $\begin{array}{c}\text { Desviación } \\
\text { estándar }\end{array}$ & Promedio & $\begin{array}{c}\text { Desviación } \\
\text { estándar }\end{array}$ & \% aumento de palabras \\
\hline A & 23 & 7,09 & 4,60 & 37,39 & 30,54 & 18,95 \\
\hline B & 21 & 10,52 & 9,99 & 25,14 & 21,04 & 41,86 \\
\hline C & 22 & 12,45 & 14,17 & 33,55 & 29,40 & 37,13 \\
\hline D & 16 & 13,56 & 26,34 & 49,00 & 42,73 & 27,68 \\
\hline Total & 82 & 10,67 & 14,74 & 35,49 & 31,48 & 30,07 \\
\hline
\end{tabular}

En el análisis cualitativo del parámetro extensión se encontró que no en todos los PMM ese aumento ocurre de la misma manera o siguiendo la misma línea de pensamiento. Se identificaron dos estrategias utilizadas por los alumnos. En la primera, los alumnos integraron la nueva información a la estructura que utilizaron en el PMM-Pre, expresando una relación de continuidad entre las informaciones mencionadas por los alumnos en el PMM-Pre y el PMMPos (figura 4). En el segundo caso, los alumnos adicionan nueva información y nuevas palabras sin dar continuidad o complementariedad a lo expresado en el PMM-Pre. La nueva información aparece desvinculada de lo mencionado antes de la visita, sin un diálogo explicito, o una interrelación evidente, una relación de discontinuidad (figura 5).

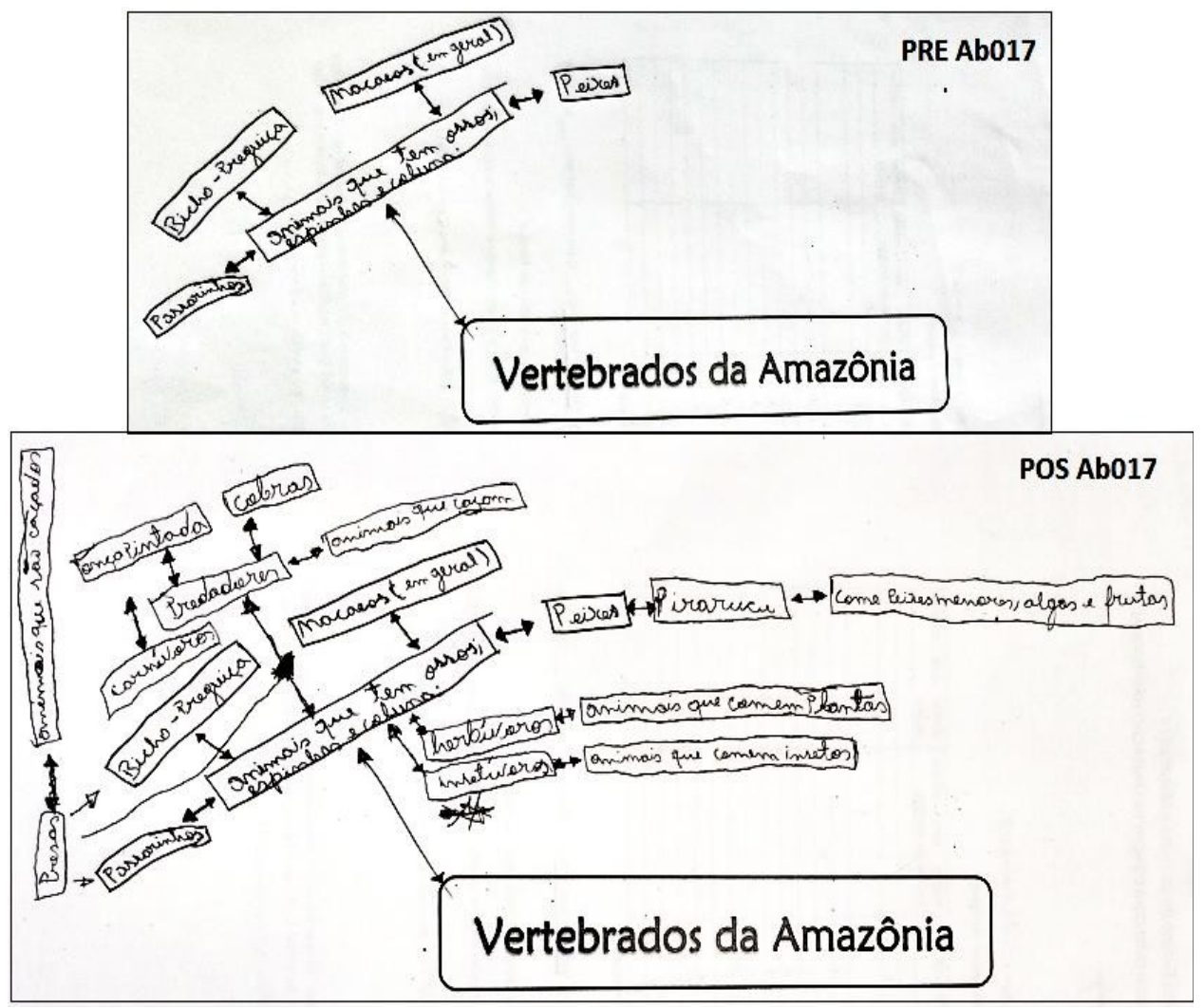

Figura 4. Relación de continuidad entre las respuestas de un alumno en el PMM-Pre y el PMM-pos. Contenido: PMM-Pre: «Vertebrados de la Amazonía: Animales que tienen huesos, espina y columna; peces, monos (en general), oso perezoso, pajaritos» PMM-Pos: «Vertebrados de la Amazonía: animales que tienen huesos, espina y columna; herbívoros, animales que comen plantas, insectívoros, animales que comen insectos; predadores: serpientes, jaguar, carnívoros; presas, animales que son cazados, pajaritos, oso perezoso; monos (en general); peces: pirarucu, come peces menores, algas y frutas». 


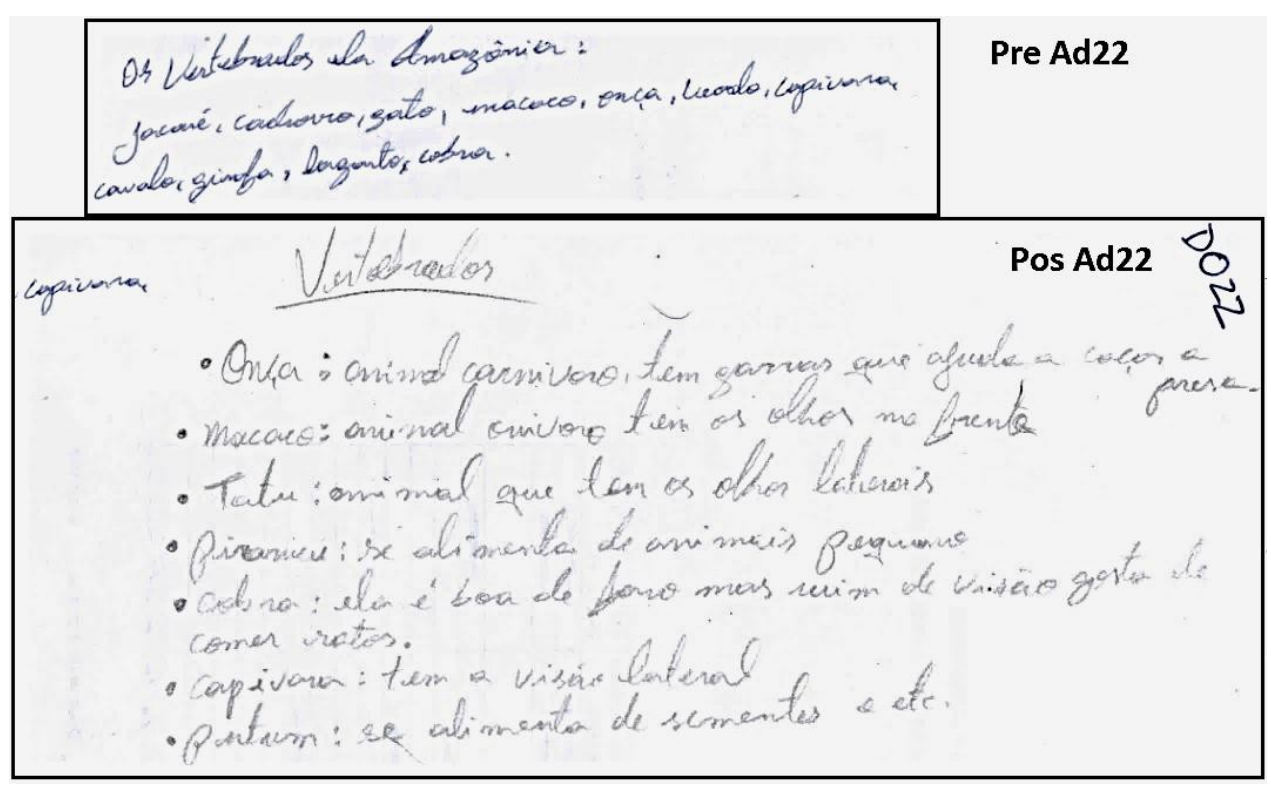

Figura 5. Relación de discontinuidad entre las respuestas de un alumno en el PMM-Pre y el PMM-Pos. Contenido: PMM-Pre: «Los vertebrados de la Amazonía: cocodrilo, perro, gato, mono, jaguar, capibara, caballo, jirafa, lagarto, serpiente». PMM-Pos: «Vertebrados: jaguar, animal carnívoro, tiene garras que le ayudan a cazar la presa; mono: animal omnívoro, tiene los ojos al frente; armadillo, animal que tiene los ojos laterales; pirarucu, se alimenta de animales pequeños; serpiente, ella es buena de [palabra no identificada] pero es pésima de visión, le gusta comer ratones; capibara, tiene visión lateral; paujil, se alimenta de semillas, etc.»

En relación al porcentaje de alumnos que utilizó cada estrategia, el 20,73 \% ( $n=17)$ presentó una relación de continuidad entre el PMM-Pre y el PMM-Pos, y el 79,27\% $(n=65)$ una relación de discontinuidad. De esta manera, el PMM se configura como una herramienta que permite identificar la relación que el alumno visitante establece entre nuevas informaciones con las que tiene acceso en la visita, con sus conocimientos previos. Este resultado plantea un desafío para las exposiciones itinerantes. El desafío consiste en planear e implementar estrategias que permitan que el visitante establezca diálogos más explícitos entre nuevas informaciones y sus conocimientos previos, considerando el corto tiempo de duración de la visita.

\section{Alcance}

A partir del análisis cualitativo de los PMM-Pre y los PMM-Pos de los alumnos, se identificaron cuatro perspectivas de explicación sobre el tema "Vertebrados de la Amazonía» por parte de los alumnos: reconocimiento, anatómica, ecológica y cultural.

Perspectiva de reconocimiento: desde esta perspectiva los alumnos reconocen que hay gran variedad de vertebrados en el bioma amazónico o realizan una lista con los nombres comunes de los que conocen (figura 6 ).

Perspectiva anatómica: perspectiva desde la cual se abordan principalmente las características anatómicas de los vertebrados en general o de un organismo en específico. Normalmente se hace alusión a la presencia de vertebras o huesos, la presencia de cráneo, la posición de las cavidades oculares, tamaño de los apéndices, presencia o no de garras, entre otros.

Perspectiva ecológica: Trata de las relaciones que los vertebrados establecen con otros seres vivos y con su medio. Desde esta perspectiva los alumnos destacan los hábitos alimenticios de determinado organismo, agrupan los organismos de acuerdo a esas características o realizan relatos o dibujos sobre el hábitat y nicho ecológico del organismo (figura 7). 


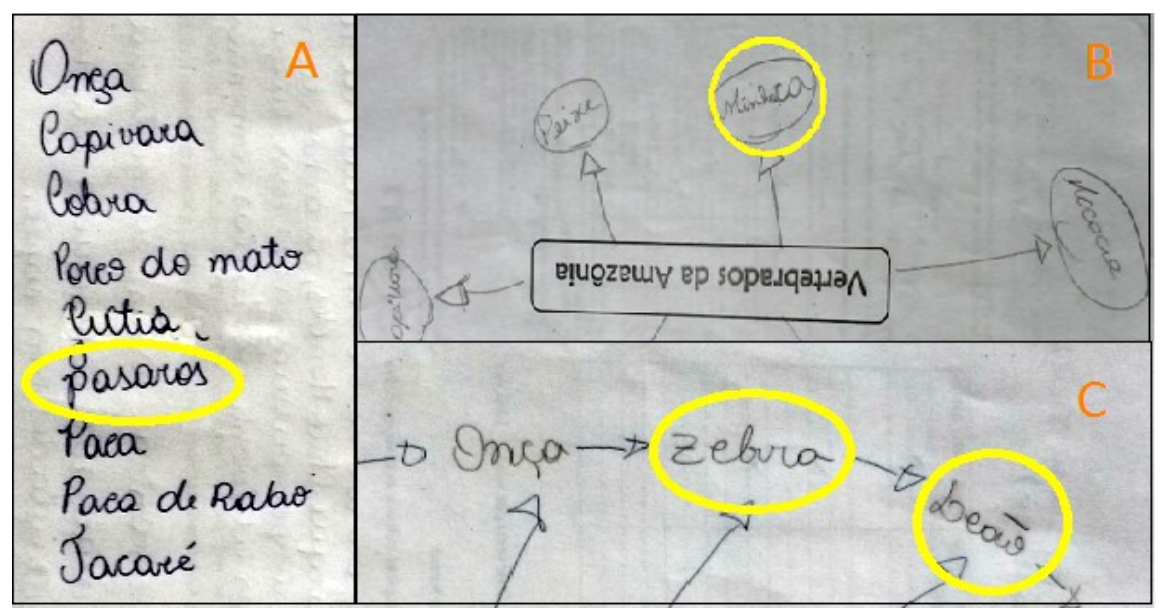

Figura 6. Perspectivas de explicación de reconocimiento en las respuestas de tres alumnos al PMM-Pre. Contenido: A) «jaguar, capibara, serpiente, puerco de monte, guatín, pájaros, paca, paca de rabo, cocodrilo.» B) «capibara, pez, lombriz, mono.» C) «jaguar, cebra, león».

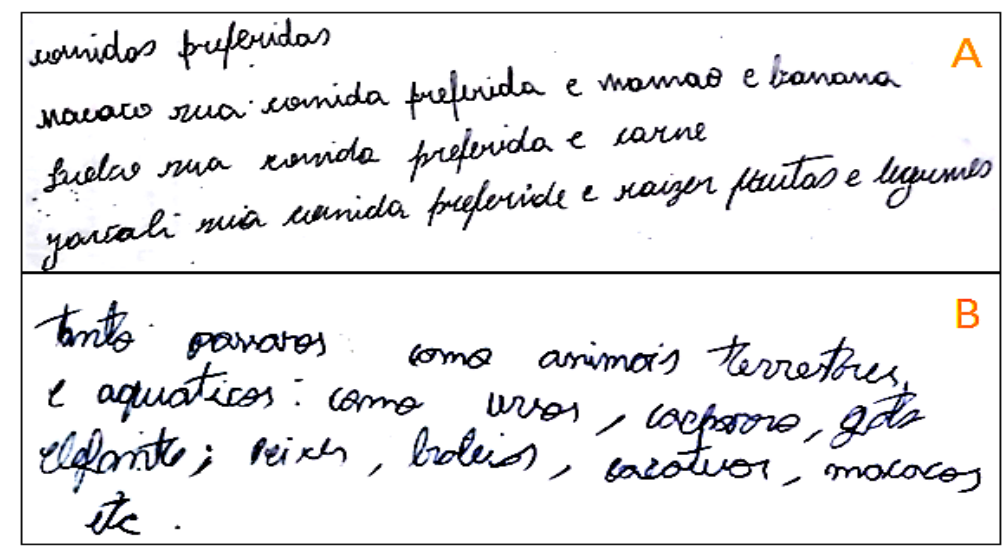

Figura 7. Perspectivas de explicación en las respuestas de dos alumnos en el PMM-Pre. Contenido: A) «Comidas preferidas; mono, su comida preferida es papaya y banana; [palabra no identificada] su comida preferida es carne; jabalí, su comida preferida es raíces, frutas y legumbres.» B) «Tanto pájaros como animales terrestres y acuáticos, como osos, perro, gato, elefante, peces, ballenas, cacatúas, monos, etc.».

Perspectiva cultural: trata de la relación del hombre con los vertebrados en el bioma amazónico. En esta perspectiva los alumnos relatan preocupación por la aceleración de los procesos de extinción de algunas de estas especies o relatan expectativas que tienen con los vertebrados, tales como poder tocarlos, conocerlos más de cerca, tenerlos como mascota.

El análisis cualitativo de las respuestas de los alumnos antes de la visita permitió identificar un aspecto que puede ser considerado positivo. Los alumnos mencionaron animales vertebrados silvestres nativos de la Amazonía (figura 6A), siendo pocos los casos de alumnos que registraron animales exóticos (figura $6 \mathrm{C}$ ). Ese aspecto difiere de los resultados de otras investigaciones en grandes centros urbanos (Bermudez et al. 2014, Orozco 2016), donde se destaca el escaso conocimiento de la biodiversidad nativa por parte de niños y jóvenes. Sin embargo, también se identificaron dos elementos que pueden ser considerados problemáticos. El primero se trata de que los alumnos reconocen con más facilidad a los mamíferos, más que a otros grupos de vertebrados (figura 6A). El segundo se refiere a que el conocimiento de la existencia de algunos vertebrados de la Amazonía no garantiza un conocimiento profundo sobre las características anatómicas, ecológicas (figura 7A y 7B) o culturales relacionadas a esos 
organismos. En algunos casos, los alumnos mencionaron animales que no son vertebrados, como la lombriz (figura 6B).

El análisis cualitativo permitió identificar las cuatro perspectivas de explicación del tema abordadas por los estudiantes, para posteriormente realizar la identificación cuantitativa del número de perspectivas abordadas por los alumnos antes y después de la visita. En el análisis cuantitativo fue identificado que en los PMM-Pre fue recurrente que los alumnos abordaran el tema «Vertebrados de la Amazonía» desde una única perspectiva. Mientras que en los PMMPos los alumnos utilizan dos o más perspectivas de explicación para referirse al mismo asunto.

En relación al porcentaje de alumnos que optaron por no mencionar nada sobre el tema, pasó del 12,2 \% $(n=10)$ antes de la visita, a 1,2 \% $(n=1)$ después de la visita. Desde una única perspectiva pasó del $62,2 \%(n=51)$ a $35,4 \%(n=29)$. Mientras que el porcentaje de alumnos que abordaron el tema desde dos o más perspectivas después de la visita aumentó. Antes de la visita el $22 \%(n=18)$ abordó el tema desde dos perspectivas, después de la visita el 37,8 \% $(n=31)$. Desde tres perspectivas ocurrió un aumento del 3,7\% $(n=3)$ al 23,2 \% $(n=19)$. Antes de la visita ninguno de los alumnos abordó el tema desde las cuatro perspectivas, después de la visita el 2,4\% $(n=2)$ de los alumnos lo consiguió.

Participar de la experiencia de la visita a la exposición contribuyó a que la mayoría de los alumnos participantes abordaran el asunto desde perspectivas que no habían contemplado en los PMM-Pre, y en algunos casos, que vincularan esas perspectivas simultáneamente. Este es un aspecto importante para el aprendizaje sobre la conservación de la biodiversidad de cualquier grupo taxonómico, en este caso los vertebrados de la Amazonía. La biodiversidad es un concepto polisémico e integrador de las diversas disciplinas de la biología y la ecología, y que además puede ser explicado desde perspectivas políticas, económicas, sociales y culturales (Orozco 2017). Por lo tanto, se evidencia que la exposición itinerante contribuyó a que los alumnos amplíen las perspectivas desde las cuales abordan la diversidad de vertebrados de la Amazonía.

En la figura 8 se presenta un ejemplo del PMM-Pos de una estudiante que brevemente abordó el tema «Vertebrados de la Amazonía» contemplando aspectos de reconocimiento, anatómicos, ecológicos y culturales.

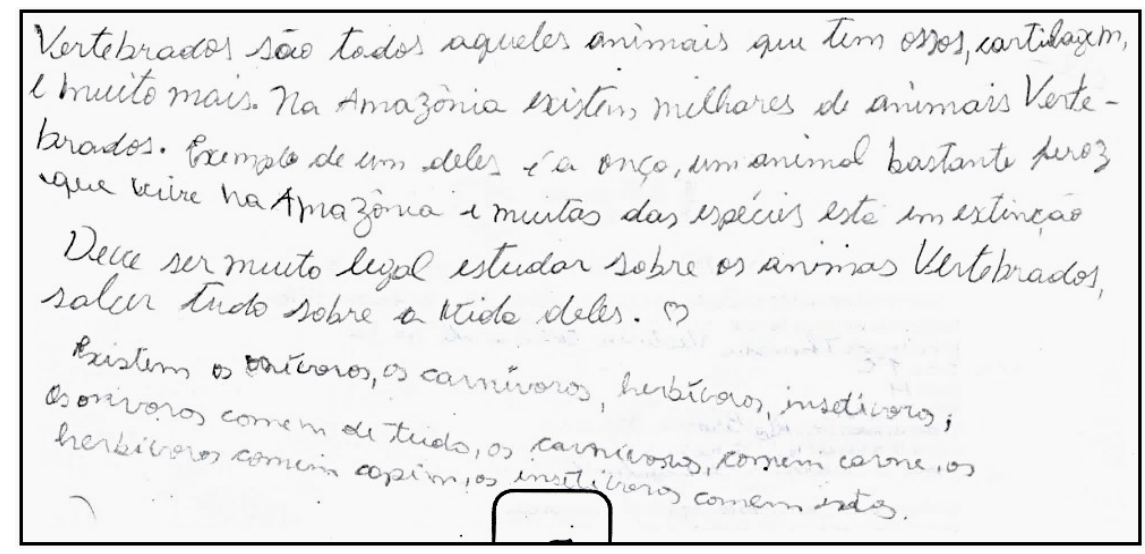

Figura 8. Perspectivas de explicación, reconocimiento, anatómica, ecológica y cultural en la respuesta de un alumno en el PMM-Pos. Contenido: Perspectiva anatómica: «Vertebrados son todos aquellos animales que tienen huesos, cartílago y mucho más». Perspectiva de reconocimiento: «En la Amazonía existen millares de animales vertebrados. Ejemplo de uno de ellos es el jaguar, bastante feroz que vive en la Amazonía» Perspectiva cultural: «Y muchas especies en extinción. Debe ser muy interesante estudiar sobre los animales vertebrados, saber todo sobre la vida de ellos». Perspectiva ecológica: «Existen los omnívoros, los carnívoros, los herbívoros, los insectívoros. Los omnívoros comen de todo, los carnívoros comen carne, los herbívoros comen pasto, los insectívoros comen insectos». 
Los resultados del parámetro alcance muestran la importancia de ampliar la comprensión sobre el aprendizaje en exposiciones itinerantes. No únicamente como el aumento de información o conceptos que un sujeto alcanza después de determinada experiencia de aprendizaje. También como la capacidad de abordar determinado fenómeno o situación desde otras perspectivas, ampliar el universo de visión sobre un asunto.

\section{Profundidad}

En este análisis fue posible identificar cuántas palabras fueron mencionadas por los alumnos en las cuatro categorías de temas identificados: reconocimiento, anatómica, ecológica y cultural. Cuantitativamente la profundidad fue abordada como la extensión dentro de cada tema. En todas las categorías hubo aumento en el número de palabras después de la visita, excepto en la perspectiva cultural (figura 9).

Los resultados muestran que en los PMM-Pre la perspectiva de reconocimiento fue abordada con mayor profundidad. Entendiendo profundidad como un mayor número de palabras mencionadas dentro de esa perspectiva, reconocimiento se destacó significativamente de las otras perspectivas antes de la visita. En la perspectiva reconocimiento, los alumnos hicieron una lista de nombres de animales que ellos reconocían como vertebrados de la Amazonía o reconocen que hay gran variedad de vertebrados en el bioma Amazónico.

Las medias de las palabras mencionadas en las perspectivas de explicación anatómica, ecológica y cultural no fueron diferentes estadísticamente. Sin embargo, la perspectiva de explicación ecológica se destacó significativamente de las otras perspectivas y presentó un aumento mayor de los PMM-Pre a los PMM-Pos. Este aspecto puede ser explicado por el hecho de que las actividades realizadas durante la visita tenían como eje temático articulador los hábitos alimentarios de los vertebrados de la Amazonía en relación con sus características anatómicas (figura 9).

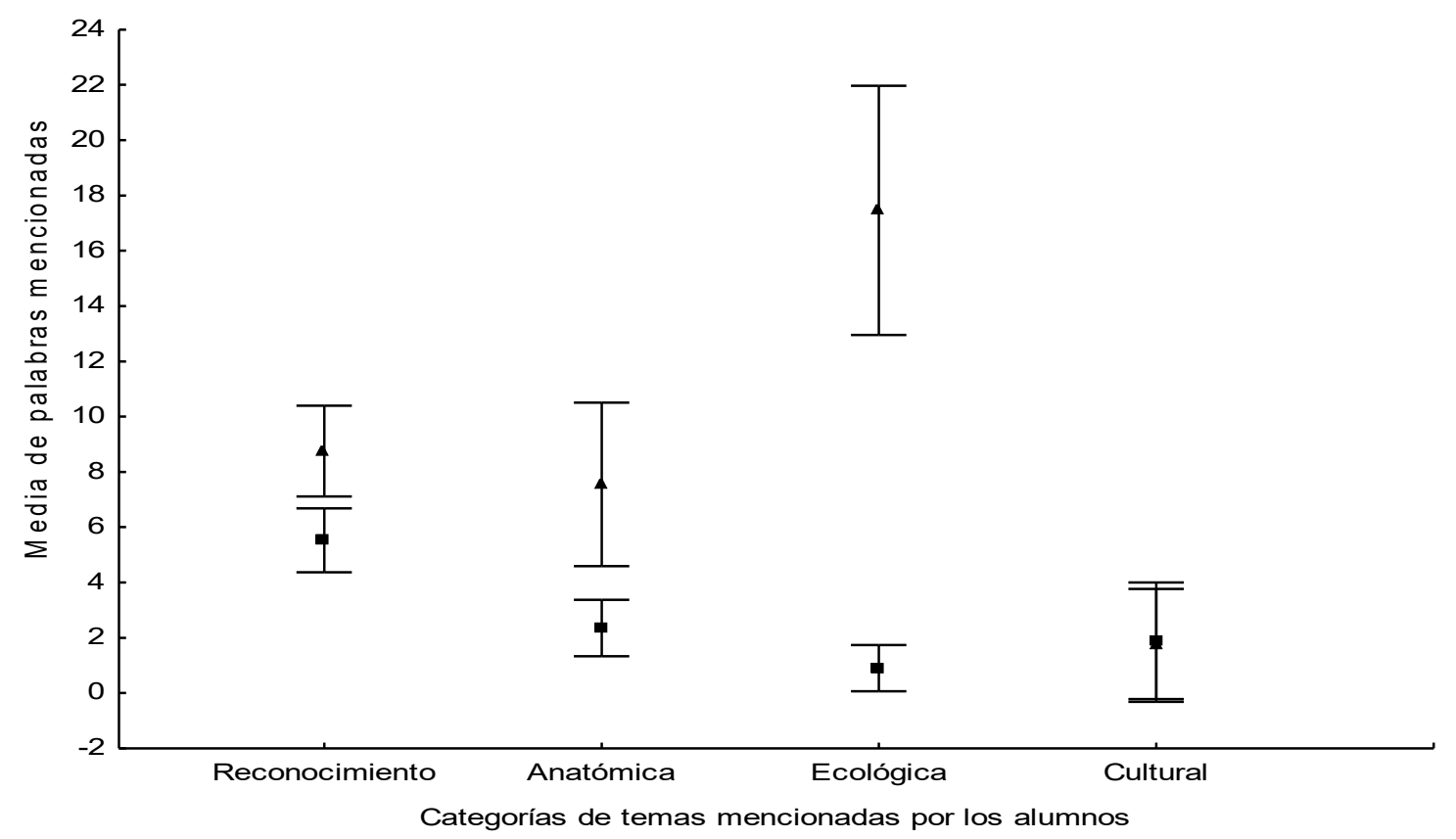

Figura 9. Media de palabras mencionadas por los alumnos dentro de cada perspectiva de explicación (Reconocimiento, Anatómica, Ecológica, Cultural) sobre los vertebrados de la Amazonía. Los cuadrados llenos representan la media en el PMM-Pre, y los triángulos la media de los PMM-Pos. Prueba de Friedman ANOVA (análisis de varianza) y Kendall chi-cuadrado $(n=82$, grados de libertad=7) $=198,8580 p<0,001$. Coeficiente de concordancia $=0,34644$, promedio rango $r=0,33837$. La no superposición de las barras representativas del intervalo de confianza de $95 \%$ indica una diferencia significativa. 
Este resultado coincide con la consideración de Rachid (2006) de que el material biológico (en este caso, las piezas anatómicas de los vertebrados), como objetos de enseñanza en los espacios no formales de educación facilitan la enseñanza y el aprendizaje. Principalmente, de los contenidos relacionados con la identificación y la clasificación de las especies, y la comprensión de los sistemas ecológicos.

Entre las categorías identificadas en las palabras mencionadas por los alumnos, la perspectiva cultural no se destacó significativamente, probablemente porque los alumnos son residentes de contexto urbano. El conocimiento de los animales de la región para la caza, fines terapéuticos y hasta como animales domésticos permanece lejos del ambiente urbano. Jonh Berger, en «Why look at animals?», relata que a partir de la Revolución Industrial se produjo la marginación de los animales en las sociedades modernas. Los animales domésticos de carga fueron sustituidos por máquinas a vapor y los animales salvajes se distanciaron por la pérdida de áreas naturales. Más tarde, en la sociedad postindustrial, los animales fueron tratados como mercancía. La exploración del mundo natural ocurrió hace mucho tiempo, pero en el siglo XIX la exploración tomó escala industrial. En el nuevo modelo de producción económica, los animales como fuente de alimento fueron tratados como commodities y manufacturados. En varias culturas, incluso en las ciudades, los animales permanecen con representación simbólica de varios aspectos de la vida cotidiana (Berger 1980, Kalof 2007).

Sin embargo, el análisis cualitativo permite identificar que este abordaje desde la perspectiva ecológica, se dio principalmente en el nivel de las especies de la biodiversidad. Con la noción de organismo como la más perceptible, específicamente en algunos vertebrados de la Amazonía. García (1997) denomina esta situación como un «énfasis en el mesocosmos», una actividad cognitiva centrada en lo perceptible e inmediato en el espacio-tiempo.

Los resultados del análisis cualitativo muestran que el nivel de pensamiento que predominó en las respuestas de los alumnos, tanto en los PMM-Pre como en los PMM-Pos, fue el nivel de la repetición, a pesar de una leve disminución del 87,8 \% $(n=72)$ de los alumnos antes de la visita, frente un 76,8\% $(n=63)$ después de la visita.

$$
\begin{aligned}
& \text { - Animair vertebrados sono aupeles que tam verterra. }
\end{aligned}
$$

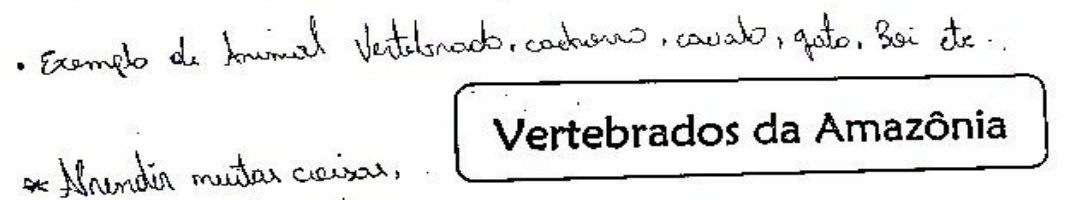

$$
\begin{aligned}
& \text { Como eles ver, duando on } \\
& \text { dhos näo laterais é Pan ter } \\
& \text { o hesse, Quando sañ os ollos from }
\end{aligned}
$$

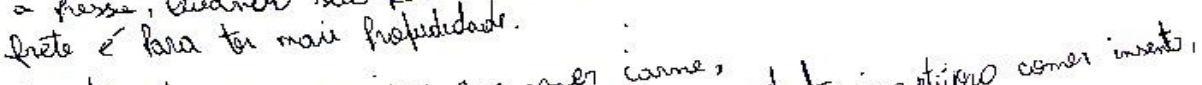

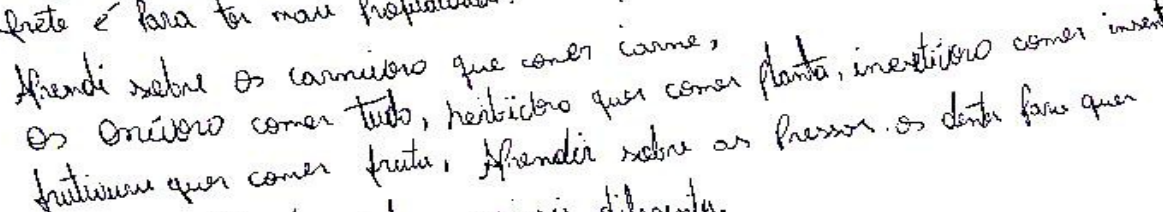

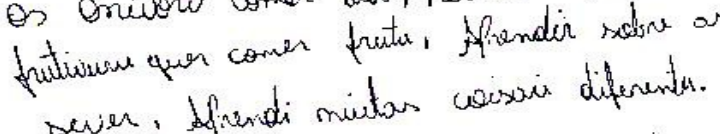

Figura 10. Aproximación al nivel de pensamiento conceptual en la respuesta de un alumno en el PMM-Pos. Contenido: PMM-Pre: «Animales vertebrados son aquellos que tienen vertebras. Ejemplo de animal vertebrado, perro, gato, caballo, gato, vaca, etc.». PMM-Pos: «Aprendí muchas cosas nuevas, como ellos ven, cuando los ojos son laterales es para ver la presa, cuando son los ojos hacia el frente es para tener mayor profundidad. Aprendí sobre los carnívoros que comen carne, los omnívoros que comen todo, herbívoros que comen planta, insectívoros comen insectos, frugívoros que comen frutas. Aprendí sobre las presas, los dientes para que sirven, aprendí muchas cosas diferentes». 
Sin embargo, en los PMM-Pre de los alumnos, ninguno alcanzó el nivel conceptual o de pensamiento estratégico, mientras que en los PMM-Pos, $22 \%(n=18)$ de los alumnos tuvieron aproximaciones a ese nivel de pensamiento. En la figura 10, el alumno utiliza conceptos como herbívoro, carnívoro, omnívoro para referirse a los hábitos alimenticios de algunos animales. De manera indirecta, el alumno se aproxima a algunas relaciones entre algunas características anatómicas con la condición de presa o predador.

Solamente el 2,4\% $(n=2)$ de los alumnos se aproximaron del nivel de pensamiento estratégico en los PMM-Pos. En la figura 11 se observa que la alumna realiza algunas breves y puntuales relaciones entre las características anatómicas de los vertebrados con sus hábitos ecológicos de alimentación. El porcentaje restante se refiere a los alumnos que no respondieron nada antes y después de la visita, ya descrito en los resultados del parámetro alcance.

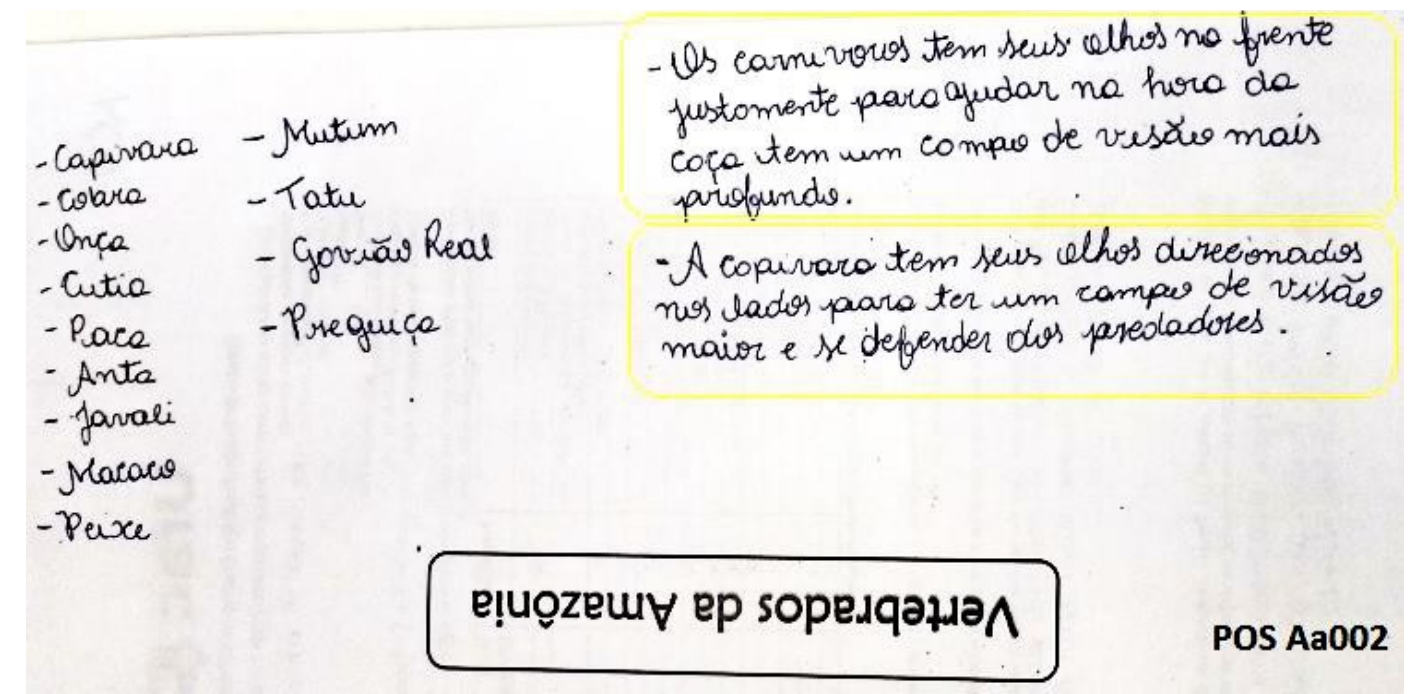

Figura 11. Aproximación al nivel de pensamiento estratégico en la respuesta de un alumno en el PMM-Pos. Contenido: PMM-Pos, parte resaltada en amarillo: «Los carnívoros tienen sus ojos al frente, justamente para ayudar al momento de la caza a tener un campo de visión más profundo. La capibara tiene sus ojos direccionados en los lados para tener un campo de visión mayor y defenderse de los predadores».

Es importante considerar que el aprendizaje es producto de la conjunción de aspectos del contexto personal, sociocultural y físico (Falk y Dierking 2002). Por lo tanto, los resultados hasta aquí mostrados no pueden atribuirse exclusivamente a las acciones de mediación en la exposición, a las actividades o a la exposición como un todo. Sin embargo, no puede excluirse la responsabilidad de la exposición en los resultados anteriores, y en estos que muestran el desafío de promover estrategias en los espacios no formales de educación que favorezcan que el visitante consiga superar la mera repetición de información, considerando aspectos como el poco tiempo de interacción con el visitante, las condiciones del espacio, la heterogeneidad de los públicos.

Este resultado se configura como retroalimentación para considerar posibles reorientaciones o modificaciones de las actividades y estrategias de enseñanza de la exposición. Por ejemplo, el uso de juegos que exigen que los sujetos realicen conexiones más explícitas entre informaciones sobre la anatomía y la ecología de los vertebrados. Pensar en procesos de formación de los mediadores, para que además de utilizar la pregunta como estrategia de mediación, pueda ser mejorada la calidad de esas preguntas. Complementar la exposición de los esqueletos y los juegos, con talleres orientados hacia el desarrollo de habilidades de argumentación y solución de problemas. Habilidades que pueden contribuir a superar la mera repetición de información como evidencia de aprendizaje por parte del público en exposiciones itinerantes. 


\section{Conclusión}

Se identificó que la exposición itinerante favoreció la construcción de aprendizajes por parte de los alumnos visitantes. El parámetro de extensión fue importante para comprobar los aspectos en el de aumento en el vocabulario y la relación de continuidad del conocimiento. El aumento en el vocabulario de los alumnos fue representado por el mayor número de palabras mencionadas por los alumnos después de la visita, principalmente desde una perspectiva ecológica. La mayoría de los alumnos no consiguieron establecer una relación de continuidad de los conocimientos, evidenciados por la repetición (nivel de pensamiento repetición) de los conocimientos. La construcción del aprendizaje de los alumnos a través de la exposición también puede percibirse por el aumento del porcentaje de alumnos que alcanzaron del nivel de pensamiento conceptual después de la visita (0 a $22 \%$ ). A partir del vocabulario adquirido en la exposición, los alumnos fueron capaces de utilizar la terminología de conceptos para describir las características de los animales de la exposición.

La integración de análisis cuantitativos y cualitativos para caracterizar las evidencias de aprendizaje permitió tener una visión más amplia sobre el aprendizaje de los alumnos. No únicamente como un producto acabado, sino también como un proceso de interacciones entre las ideas previas del sujeto, el diálogo en la exposición y la interacción con los objetos que la conforman. Los resultados de esta caracterización permiten destacar el potencial de las exposiciones itinerantes para contribuir con el aprendizaje de público escolar sobre la biodiversidad local. Sugiriendo un desafío para las exposiciones: fortalecer los procesos de enseñanza y de aprendizaje, como espacios de educación no formal, aliados de los contextos formales.

\section{Agradecimientos}

Nuestros agradecimientos especiales a la Universidad Federal del Acre, a la Organización de los Estados Americanos (OEA), a la Coordinación de perfeccionamiento de personal de nivel superior (CAPES) y al Consejo Nacional de desarrollo científico y tecnológico de Brasil (CNPQ) por el apoyo logístico y financiero.

\section{Referencias}

Belia S., Fidler F., Williams J., Cumming G. (2005) Researchers Misunderstand Confidence Intervals and Standard Error Bars. Psychological Methods 10 (4), 389-396.

Bermudez G., García M., Batistón L., Diaz S., De Longhi A. (2014) El conocimiento de las especies vegetales nativas de estudiantes de escuela media en Córdoba, Argentina. Memorias X Jornadas Nacionales $V$ Congreso Internacional de Enseñanza de la Biología. Bariloche, Argentina.

Berger J. (1980) Why look at animals? pp. 1-26 en About Looking. New York: Pantheon.

Bizerra A., Marandino M. (2009) A concepção de “aprendizagem” nas pesquisas em educação em museus de ciências. Anais VII Encontro de Pesquisa em Educação em Ciências. Florianópolis.

Creswell J., Garret A. (2008) The "movement" of mixed methods research and the role of educators. South African Journal of Education 28 (3), 321-333.

Cumming G., Fidler F. Vaux D. (2007). Error bars in experimental biology. The Journal of Cell Biology 177 (1), 7-11.

Delicado A., Cortez A., Vala F., Do Mar M., Casaleiro P. (2010) Comunicar ciência numa exposição: uma avaliação exploratória de a evolução de Darwin através de PMM. Actas I Seminário de Investigação em Museologia dos Países de Lingua Portuguesa e Espanbola 2, 8-18. 
Falk J., Dierking L. D. (2002) Lessons Without Limit - How free-choice learning is transforming education. California: Altamira Press.

Falk J., Storksdieck M. (2005) Using the Contextual Model of Learning to Understand Visitor Learning from a Science Center Exhibition. Science Education 89 (5), 744-778.

Friedman M. (1940). A comparison of alternative tests of significance for the problem of $\mathrm{m}$ rankings. The Annals of Mathematical Statistics 11 (1), 86-92.

García J. (1997) Una hipótesis de progresión sobre los modelos de desarrollo en educación ambiental. Investigación en la Escuela 37, 15-32.

Johnson R., Onwuegbuzie A. (2004) Mixed Methods Research: A Research Paradigm Whose Time Has Come. Educational Researcher 33 (7), 14-26.

Kalof L. (2007) Looking at animals in buman history. London: Reakion Books.

Kruskal W. H., Wallis W. A. (1952). Use of ranks in one-criterion variance analysis. Journal of the American Statistical Association 47 (260), 583-621.

Leinhardt G., Crowley K., Knutson K. (2002) Learning conversations in museums. Mahwah, NJ: Lawrence Erlbaum.

Marandino M. (org.), Fernandes A., Navas A., Contier D., Standerski L., Magalhaes L., Conrado L., Correia M., Rachid V. (2008) Educação em Museus: A mediação em focos. São Paulo: FEUSP.

Menin M. (2010) Amazônia: diversidade biológica e bistória geológica. Módulo Regionalizado 3. Goiânia: FUNAPE/Universidade Federal de Goiás.

Moss A., Jensen E., Gusset M. (2014) Evaluating the contribution of zoos and aquariums to Aichi Biodiversity Target 1. Conservation Biology 29 (2), 537-544.

Orozco Y. (2016) Relação escola-território no ensino do conceito biodiversidade. O que os alunos de um contexto rural colombiano sabem sobre a biodiversidade que os rodeia. Anais X Simpósio Linguagens e identidades da/ na Amazônia Sul-Ocidental. Rio Branco Brasil.

Orozco Y. (2017) O ensino da biodiversidade: tendências e desafios nas experiências pedagógicas. Góndola, Enseñanza y Aprendizaje de las Ciencias 12 (2), 173-185.

Peruquetti P., Kassab S., Santos, B., Mendonça, E., Carvalho Y. (2018) Perfil de visitantes em exposição científica na Semana Nacional de Ciência e Tecnologia na região norte do Brasil. South American Journal of Basic Education, Technical and Technological 5 (1), 113-124.

Rachid V. (2006) O processo de aprendizagem no Zôo de Sorocaba: Análise da atividade educativa visita orientada a partir dos objetos biológicos. Dissertação, Mestrado em Educação. Faculdade de Educação, Universidade de São Paulo. São Paulo, Brasil.

Rocha J. N., Marandino M. (2017) Mobile science museums and centers and their history in the public communication of science. The Journal of Science communication 16 (03), A04.

Roland C., Vasconcelos C. (2007) Biodiversidade Amazônica: valor, potencialidades e riscos, pp. 127-152 en Val Adalberto L., Santos Geraldo M. (orgs.). Grupo de Estudos Estratégicos Amazônicos, Caderno de Debates, Tomo I. INPA, Manaus.

Roldan A., Ulloa D., Vargas L., Chura Z., Pacheco F. (2017) Comparación entre recorridos guiados tradicionales y recorridos guiados indagatorios en el Museo Nacional de Historia Natural, La Paz-Bolivia. Revista Eureka sobre Enseñanza y Divulgación de las Ciencias 14 (2), 367-384. 
Sanches A., Leite J., Leroy P. (2011) Aprendizagem no Planetário: Concepções e Conhecimentos Adquiridos por Alunos do Ensino Fundamental. Atas VIII Encontro Nacional de Pesquisa em Educação em Ciências - VIII ENPEC. Aguas de Lindóia.

Schwenck B. (2011) Ciência móvel: A mediação informacional nas exposições de um museu itinerante. Dissertação (Mestrado em Ciência da Informação)- Rio de Janeiro, IBICT, UFRJ/FAZZ, Rio de Janeiro.

Soler M. G., Landim M.I. (2017) O silêncio dos inocentes: o papel dos animais em narrativas expositivas. Anais do Museu Paulista 25 (2), 269-289.

Villa M. (2016) Learning at the Science Museum. A study on the public's experiences with different types of visit at the Museo Nazionale della Scienza e della Tecnologia "Leonardo da Vinci" in Milan, Italy. Journal of Science Communication 15 (04), A01.

Webb N., Alt M., Ely R., Vesperman B. (2005) Web Alignment Tool (WAT) Training Manual. Madison, WI, U.S.A.: Wisconsin Center for Education Research. 